\title{
Erratum:
}

\section{Erratum to: RegIV expression showing specificity to gastrointestinal tract and its potential role in diagnosing digestive tract neuroendocrine tumor}

\author{
Feng-ying $\mathrm{LI}^{1,2}$, Xiao-bin $\mathrm{REN}^{3}$, En-ping XU⿱1${ }^{1}$, Qiong HUANG ${ }^{1}$, \\ Hong-qiang SHENG ${ }^{1}$, Bing-jian $\mathrm{LV}^{4}$, Mao-de LAI ${ }^{\dagger 1}$ \\ $\left({ }^{1}\right.$ Department of Pathology, School of Medicine, Zhejiang University, Hangzhou 310058, China) \\ $\left({ }^{2}\right.$ Clinical Laboratory, Sir Run Run Shaw Hospital, School of Medicine, Zhejiang University, Hangzhou 310016, China) \\ ( ${ }^{3}$ Department of Epidemiology and Biostatistics, School of Medicine, Zhejiang University, Hangzhou 310058, China) \\ $\left({ }^{4}\right.$ Anatomical and Cellular Pathology, Women’s Hospital, School of Medicine, Zhejiang University, Hangzhou 310006, China) \\ ${ }^{\dagger}$ E-mail: lmd@zju.edu.cn
}

doi:10.1631/jzus.B09e0383 Published online: Apr. 10, 2010

\section{Erratum to: J Zhejiang Univ-Sci B (Biomed \& Biotechnol) doi:10.1631/jzus.B0900383}

The original version of this article unfortunately contained a mistake. The presentation of Table 6 was missing. The table is given below:

Table 6 RegIV expression in neuroendocrine tumors

\begin{tabular}{lcc}
\hline \multicolumn{1}{c}{ Neuroendocrine tumors } & RegIV & CgA \\
\hline Digestive system & & $5 / 5$ \\
Gastric carcinoid & $3 / 5 ; 1(+), 2(+++) ; \mathrm{PN}$ & $\mathrm{NA}$ \\
Gastric NEC & $1 / 2 ;(+++) ; \mathrm{M} / \mathrm{PN}$ & $1 / 1$ \\
Small intestinal carcinoid & $0 / 1$ & $3 / 4$ \\
Colorectal carcinoid & $4 / 4 ; 1(+), 3(+++) ; \mathrm{PN}$ & $5 / 6$ \\
Appendiceal carcinoid & $5 / 6 ; 1(+), 4(+++) ; \mathrm{PN}$ & $5 / 5$ \\
Islet cell tumors of pancreas & $0 / 5$ & $\mathrm{NA}$ \\
Pancreas NEC & $1 / 1 ;(+++) ; \mathrm{C}$ & \\
Non-digestive system & & $1 / 3$ \\
Lung typical carcinoid & $0 / 3$ & $5 / 5$ \\
Lung atypical carcinoid & $0 / 5$ & $5 / 7$ \\
Small cell lung carcinoma & $0 / 7$ & $1 / 2$ \\
Large cell lung carcinoma & $0 / 2$ & $\mathrm{NA}$ \\
Lung NEC & $0 / 2$ & $4 / 5$ \\
Pituitary adenoma & $0 / 5$ & $5 / 5$ \\
Medullary thyroid carcinoma & $2 / 5 ; 2(+) ; \mathrm{C}$ & $6 / 6$ \\
Parathyroid adenoma & $0 / 6$ & $5 / 5$ \\
Thymic carcinoid & $0 / 5$ & $7 / 8$ \\
Pheochromocytoma & $0 / 5$ & $0 / 5$ \\
Adrenal cortical adenoma & $5 / 8 ; 2(+++), 2(++), 1(+) ; \mathrm{C}$ & \\
\hline
\end{tabular}

NEC: neuroendocrine carcinoma; C: cytoplasmic staining; PN: perinuclear staining; M: mucin-like staining; NA: results not available 